Inequalities for the De Pril approximation to the distribution of the number of policies with claims

Raluca Vernic; Jan Dhaene; Bjorn Sundt

DEPARTMENT OF ACCOUNTANCY, FINANCE AND INSURANCE (AFI) 


\title{
Inequalities for the De Pril approximation to the distribution of the number of policies with claims
}

\author{
Raluca Vernic \\ Ovidius University of Constanta \\ Jan Dhaene \\ Katholieke Universiteit Leuven \\ Bjørn Sundt \\ Oslo
}

February 3, 2009

\begin{abstract}
In the present paper, we give sufficient conditions for an ordering of De Pril approximations of the distribution of the number of claims in an insurance portfolio of independent policies. Possible extensions are discussed, both for the De Pril approximation and the Kornya approximation. A numerical example is given.
\end{abstract}

\section{Introduction}

De Pril (1986) introduced a method for recursive evaluation of the aggregate claims distribution in the individual life model with independent policies with non-negative integer-valued sums assured. As this method was rather timeconsuming, Vandebroek \& De Pril (1988) and De Pril (1988) introduced an approximation by including the $r$ first terms in some summations. De Pril (1988) deduced an error bound for this approximation; we call $r$ the order of the approximation. The approximation and the error bound were extended to non-degenerate claim amount distributions on the non-negative integers by De Pril (1989).

The Kornya approximation is proportional to the De Pril approximation with proportionality factor chosen such that the approximation of the probability function sums to one like an exact probability function. This approximation was introduced by Kornya (1983) for the individual life model 
with independent policies with non-negative integer-valued sums assured and extended to non-degenerate claim amount distributions on the non-negative integers by Hipp (1986); both these authors deduced error bounds.

Dhaene \& De Pril (1994) unified the deduction of the approximations of De Pril and Kornya, as well as an approximation introduced by Hipp (1996), and error bounds for these approximations.

For probability distributions on the non-negative integers with a positive mass at zero, Sundt (1995) named a central transform in the recursions of De Pril (1986) the De Pril transform. Dhaene \& Sundt (1998) extended the definition of the De Pril transform to functions on the non-negative integers with a positive mass at zero and discussed approximations within this framework.

Sundt (2002) and Sundt \& Vernic (2009) give surveys of the theory of recursions for aggregate claims distributions. Dhaene et al. (2006) and Sundt \& Vernic (2006) compare recursions for aggregate claims distributions within an individual setting.

Numerical examples presented by Vandebroek \& De Pril (1988) and Sundt \& Vernic (2009) seem to indicate that for the cumulative distribution, for $r=1,2, \ldots$, the De Pril approximation of order $2 r-1$ is decreasing in $r$, whereas the approximation of order $2 r$ is increasing in $r$. For the Kornya approximation, the situation seems to be the opposite, the approximation of order $2 r-1$ increasing in $r$ and the approximation of order $2 r$ decreasing in $r$.

For the Kornya approximation in the individual life model, Kornya (1983) gave conditions for this property. However, in published discussions to that paper, David C. McIntosh and Donald P. Minassian pointed out an error in the proof. The latter discussant gave counterexamples.

The purpose of the present paper is to give sufficient conditions for the ordering property of the De Pril approximation to hold for the distribution of the number of policies with claims. In themselves, these results are perhaps not too interesting as in this simple case it would not be that complicated to evaluate the exact distribution, but we hope that the results can be stepstones to more general results. When each policy can have at most one claim, the distribution of the number of policies with claims can be interpreted as the claim number distribution, that is, an aggregate claims distribution with all claim amount distributions concentrated in one.

In Section 2, we introduce some notation that we shall apply in this paper. As the De Pril transform will be a central tool in our deductions, we define it and recapitulate some of its properties in Section 3. Section 4 is devoted to the relation between De Pril transforms and generating functions. In Section 5. we introduce the approximations of De Pril and Kornya. The core of our 
paper is Section 6. That is where we prove the inequalities. In Section 7 , we discuss some possible extensions, both for the De Pril approximation and the Kornya approximation. Finally, in Section 8, we present a numerical example based on a dataset introduced by Gerber (1979). This dataset has also been applied for numerical examples by Jewell \& Sundt (1981), Chan (1984), Sundt (1985), Hipp (1986), Vandebroek \& De Pril (1988), Kuon et al. (1993), Dhaene \& Goovaerts (1997), and Sundt \& Vernic (2009).

For proofs of the results in Sections 3 and 5 , see e.g. Sundt \& Vernic (2009).

\section{Notation and conventions}

In this paper, we shall study distributions on the non-negative integers. We denote the probability function by a lower-case letter, the cumulative distribution function by the corresponding capital, and the tail by a bar on that capital. Thus, if $f$ is a probability function on the non-negative integers, then

$$
F(x)=\sum_{y=0}^{x} f(y) ; \quad \bar{F}(x)=\sum_{y=x+1}^{\infty} f(y)=1-F(x) . \quad(x=0,1,2, \ldots)
$$

We use this notation also for approximations to distributions. These approximations do not necessarily sum to one, so that the identity

$$
\sum_{y=x+1}^{\infty} f(y)=1-F(x) \quad(x=0,1,2, \ldots)
$$

does not necessarily hold. In Section 8, we shall discuss the orderings mentioned in Section 1 in connection with a numerical example. When displaying the cumulative distribution function and its approximations, it is difficult to see what is going on far out in tail as the values will be close to one. Hence, it is more informative to display the tail. When defining a tail by the second expression in (1), the orderings will be preserved with the inequalities the opposite way. However, the orderings will not necessarily be preserved with the first expression if the approximations sum to different values. Hence, for convenience, we define the tail of $F$ by $\bar{F}=1-F$. We introduce $F(\infty)=\lim _{x \uparrow \infty} F(x)$ and $\bar{F}(\infty)=\lim _{x \uparrow \infty} \bar{F}(x)$ under the assumption that these limits exist.

We shall to a large extent apply De Pril transforms. As these transforms are normally more convenient to apply on probability functions than 
on cumulative distribution functions, we shall usually mean the probability function when referring to a distribution.

Let $\mathcal{P}_{0}$ denote the class of distributions on the non-negative integers with a positive mass at zero, $\mathcal{P}_{+}$the class of distributions on the non-negative integers, and $\mathcal{F}_{0}$ the class of functions on the non-negative integers with a positive mass at zero.

We denote a compound distribution with counting distribution $p$ and severity distribution $h$ by $p \vee h$. If $p \in \mathcal{P}_{0}$ and $h \in \mathcal{P}_{+}$, we have

$$
(p \vee h)(x)=\sum_{n=0}^{x} p(n) h^{n *}(x) . \quad(x=0,1,2, \ldots)
$$

Thus, $p \vee h \in \mathcal{P}_{0}$. More generally, we apply (2) as definition of the compound function $p \vee h$ when $p \in \mathcal{F}_{0}$ and $h \in \mathcal{P}_{+}$; in that case, $p \vee h \in \mathcal{F}_{0}$.

A distribution $p \in \mathcal{P}_{0}$ is the Bernoulli distribution $\operatorname{Bern}(\pi)$ if it is given by

$$
p(1)=1-p(0)=\pi . \quad(0<\pi<1)
$$

We introduce the indicator function $I$, that is, $I(A)=1$ if a condition $A$ is satisfied, and $I(A)=0$ if it is not satisfied.

For a function $f$ on the integers, we let $\Delta f(x)=f(x)-f(x-1)$ for all integers $x$. We have $f=\Delta F$.

We denote the generating function of a function $f$ on the non-negative integers by $\tau_{f}$, that is, $\tau_{f}(s)=\sum_{x=0}^{\infty} f(x) s^{x}$. When using generating functions, it is tacitly assumed that they exist.

If $x$ is a real number, then we let $[x]$ denote the largest integer less than or equal to $x$.

A summation $\sum_{j=a}^{b}$ is assumed to be equal to zero when $b<a$.

When giving a function an argument outside the range for which the function has been defined, we tacitly assume that the value of the function for that argument is equal to zero.

\section{The De Pril transform}

Inspired by De Pril (1989), Sundt (1995) defined the De Pril transform $\varphi_{f}$ of a distribution $f \in \mathcal{P}_{0}$ by the recursion

$$
\varphi_{f}(x)=\frac{1}{f(0)}\left(x f(x)-\sum_{y=1}^{x-1} \varphi_{f}(y) f(x-y)\right) \quad(x=1,2, \ldots)
$$


and studied some of its properties. Allowing for approximations to distributions, Dhaene \& Sundt (1998) extended this definition to functions in $\mathcal{F}_{0}$ and studied the properties of the De Pril transform within that wider context.

As a distribution sums to one, the De Pril transform of a distribution in $\mathcal{P}_{0}$ determines the distribution uniquely, whereas the De Pril transform of a function in $\mathcal{F}_{0}$ determines the function only up to a multiplicative constant, that is, all functions in $\mathcal{F}_{0}$ proportional to that function have the same De Pril transform.

Solving (4) for $f(x)$ gives

$$
f(x)=\frac{1}{x} \sum_{y=1}^{x} \varphi_{f}(y) f(x-y), \quad(x=1,2, \ldots)
$$

by which we can evaluate $f$ recursively if $f(0)$ is known.

It can be shown that if $f=*_{j=1}^{m} f_{j}$ with $f_{j} \in \mathcal{F}_{0}$ for $j=1,2, \ldots, m$, then

$$
\varphi_{f}=\sum_{j=1}^{m} \varphi_{f_{j}} .
$$

Thus, we can evaluate $f$ by first evaluating each $\varphi_{f_{j}}$ recursively by (4), then $\varphi_{f}$ by (6), and, finally, $f$ recursively by (5). This is De Pril's first method.

If $f=p \vee h$ with $p \in \mathcal{F}_{0}$ and $h \in \mathcal{P}_{+}$, then

$$
\varphi_{f}(x)=x \sum_{y=1}^{x} \frac{\varphi_{p}(y)}{y} h^{y *}(x) . \quad(x=1,2, \ldots)
$$

In particular, if $p$ is the Bernoulli distribution Bern $(\pi)$ given by (3), then

$$
\varphi_{p}(y)=-\left(\frac{\pi}{\pi-1}\right)^{y}, \quad(y=1,2, \ldots)
$$

so that

$$
\varphi_{f}(x)=-x \sum_{y=1}^{x} \frac{1}{y}\left(\frac{\pi}{\pi-1}\right)^{y} h^{y *}(x) . \quad(x=1,2, \ldots)
$$

Any distribution $f \in \mathcal{P}_{0}$ can be expressed as a compound distribution with counting distribution being the Bernoulli distribution Bern $(\pi)$ with $\pi=1-f(0)$ and severity distribution $h \in \mathcal{P}_{+}$given by $h(y)=f(y) / \pi$ for $y=1,2, \ldots$. Hence, instead of evaluating each $\varphi_{f_{j}}$ recursively by (4) in De Pril's first method, we can use (9). We then obtain De Pril's second method. 
De Pril's first and second methods were presented by De Pril (1986) in the individual life model and extended to distributions in $\mathcal{P}_{0}$ by De Pril (1989).

For $f \in \mathcal{F}_{0}$, Dhaene et al. (1999) showed that

$$
\varphi_{F}(x)=1+\varphi_{f}(x) . \quad(x=1,2, \ldots)
$$

Combining this with (5) gives the recursion

$$
F(x)=\frac{1}{x} \sum_{y=1}^{x}\left(1+\varphi_{f}(y)\right) F(x-y), \quad(n=1,2, \ldots)
$$

from which we see that if $\varphi_{f} \geq-1$, then $F \geq 0$.

\section{Generating functions}

For $f \in \mathcal{F}_{0}$, we obtain from (5) that

$$
x f(x)=\sum_{y=1}^{x} \varphi_{f}(y) f(x-y) . \quad(x=0,1,2, \ldots)
$$

Multiplication by $s^{x}$ and summation over $x$ gives

$$
s \tau_{f}^{\prime}(s)=\tau_{\varphi_{f}}(s) \tau_{f}(s),
$$

that is,

$$
\frac{d}{d s} \ln \tau_{f}(s)=\frac{\tau_{\varphi_{f}}(s)}{s}=\sum_{x=1}^{\infty} \varphi_{f}(x) s^{x-1} .
$$

By integration, we obtain

$$
\ln \tau_{f}(s)-\ln \tau_{f}(0)=\sum_{x=1}^{\infty} \frac{\varphi_{f}(x)}{x} s^{x},
$$

so that

$$
\tau_{f}(s)=f(0) \exp \left(\sum_{x=1}^{\infty} \frac{\varphi_{f}(x)}{x} s^{x}\right) .
$$

Letting $s=1$ gives

$$
F(\infty)=\tau_{f}(1)=f(0) \exp \left(\sum_{x=1}^{\infty} \frac{\varphi_{f}(x)}{x}\right) \geq 0 .
$$

At first glance, this seems to give the impression that $F(\infty)$ is positive for all functions $f \in \mathcal{F}_{0}$. However, we have made the convention that when applying generating functions, it is tacitly assumed that they exist. Hence, (12) indicates that $\sum_{x=1}^{\infty} \varphi_{f}(x) / x$ does not exist when $F(\infty)<0$. 
Theorem 1. Let $p=*_{j=1}^{m} p_{j}$ and $f=*_{j=1}^{m}\left(p_{j} \vee h_{j}\right)$ with $h_{j} \in \mathcal{P}_{+}, p_{j} \in \mathcal{F}_{0}$, and $\sum_{n=1}^{\infty}\left|\varphi_{p_{j}}(n)\right| / n$ convergent for $j=1,2, \ldots, m$. Then $F(\infty)=P(\infty)$.

Proof. By application of (6) and (7),we obtain

$$
\begin{aligned}
& \sum_{x=1}^{\infty} \frac{\varphi_{f}(x)}{x}=\sum_{x=1}^{\infty} \frac{1}{x} \sum_{j=1}^{m} \varphi_{p_{j} \vee h_{j}}(x)=\sum_{x=1}^{\infty} \sum_{j=1}^{m} \sum_{y=1}^{x} \frac{\varphi_{p_{j}}(y)}{y} h_{j}^{y *}(x)= \\
& \sum_{y=1}^{\infty} \frac{1}{y} \sum_{j=1}^{m} \varphi_{p_{j}}(y) \sum_{x=y}^{\infty} h_{j}^{y *}(x)=\sum_{y=1}^{\infty} \frac{1}{y} \sum_{j=1}^{m} \varphi_{p_{j}}(y)=\sum_{y=1}^{\infty} \frac{\varphi_{p}(y)}{y} .
\end{aligned}
$$

Together with 12 , this gives

$$
F(\infty)=f(0) \exp \left(\sum_{x=1}^{\infty} \frac{\varphi_{f}(x)}{x}\right)=p(0) \exp \left(\sum_{y=1}^{\infty} \frac{\varphi_{p}(y)}{y}\right)=P(\infty) .
$$

Q.E.D.

By solving (12) for $f(0)$, we obtain

$$
f(0)=\tau_{f}(1) \exp \left(-\sum_{x=1}^{\infty} \frac{\varphi_{f}(x)}{x}\right)=F(\infty) \exp \left(-\sum_{x=1}^{\infty} \frac{\varphi_{f}(x)}{x}\right) .
$$

When $f$ sums to one, like when $f \in \mathcal{P}_{0}$, this gives

$$
f(0)=\exp \left(-\sum_{x=1}^{\infty} \frac{\varphi_{f}(x)}{x}\right) .
$$

By application of 10 , we obtain that

$$
\begin{aligned}
& \tau_{F}(s)=F(0) \exp \left(\sum_{x=1}^{\infty} \frac{\varphi_{F}(x)}{x} s^{x}\right)=f(0) \exp \left(\sum_{x=1}^{\infty} \frac{1+\varphi_{f}(x)}{x} s^{x}\right)= \\
& f(0) \exp \left(-\ln (1-s)+\sum_{x=1}^{\infty} \frac{\varphi_{f}(x)}{x} s^{x}\right)
\end{aligned}
$$

when $|s|<1$. Thus,

$$
\tau_{F}(s)=\frac{f(0) \exp \left(\sum_{x=1}^{\infty} \frac{\varphi_{f}(x)}{x} s^{x}\right)}{1-s}=\frac{\tau_{f}(s)}{1-s} .
$$


Furthermore,

$$
\tau_{\bar{F}}(s)=\sum_{x=0}^{\infty} \bar{F}(x) s^{x}=\sum_{x=0}^{\infty}(1-F(x)) s^{x}=\frac{1}{1-s}-\tau_{F}(s),
$$

and insertion of (14) gives

$$
\tau_{\bar{F}}(s)=\frac{1-f(0) \exp \left(\sum_{x=1}^{\infty} \frac{\varphi_{f}(x)}{x} s^{x}\right)}{1-s}=\frac{1-\tau_{f}(s)}{1-s} .
$$

\section{Approximations}

For $f=p \vee h$ with $p \in \mathcal{P}_{0}$ and $h \in \mathcal{P}_{+}$, evaluating $\varphi_{f}(x)$ by $(7)$ can be rather time-consuming for large $x$. Hence, one often approximates $p$ with a function $p^{(r)} \in \mathcal{F}_{0}^{(r)}$ for some positive integer $r$ with $\mathcal{F}_{0}^{(r)}$ denoting the class of functions $q \in \mathcal{F}_{0}$ for which $\varphi_{q}(y)=0$ for all $y>r$.

We see that the convolution of functions in $\mathcal{F}_{0}^{(r)}$, is also in $\mathcal{F}_{0}^{(r)}$.

From $(12)$, we obtain that

$$
P^{(r)}(\infty)=p^{(r)}(0) \exp \left(\sum_{n=1}^{r} \frac{\varphi_{p^{(r)}}(n)}{n}\right)
$$

that is, the summation in the exponent has now a finite number of terms. This implies that $P^{(r)}(\infty)$ will always exist and be positive. When $P^{(r)}(\infty)=$ 1 , we get

$$
p^{(r)}(0)=\exp \left(-\sum_{n=1}^{r} \frac{\varphi_{p^{(r)}}(n)}{n}\right) .
$$

Application of (11) gives the recursion

$$
P^{(r)}(n)=\frac{1}{n} \sum_{i=1}^{n}\left(1+\varphi_{p^{(r)}}(i)\right) P^{(r)}(n-i) . \quad(n=1,2, \ldots)
$$

Here we have to sum up to $n$ although $\varphi_{p^{(r)}}(i)=0$ when $i>r$. From Corollary 8.1 in Sundt \& Vernic (2009), we obtain the alternative recursion

$$
P^{(r)}(n)=P^{(r)}(n-1)+\frac{1}{n} \sum_{i=1}^{r+1} \Delta \varphi_{p^{(r)}}(i) P^{(r)}(n-i), \quad(n=1,2, \ldots)
$$

where we avoid that problem. 
We shall now concentrate on two classes of such approximations that satisfy the condition

$$
\varphi_{p^{(r)}}(y)=I(y \leq r) \varphi_{p}(y) . \quad(y=1,2, \ldots)
$$

This condition determines the approximation only up to a multiplicative constant, so that we need another condition to determine that constant:

1. For the De Pril approximation, we let

$$
p^{(r)}(0)=p(0) \text {. }
$$

It is immediately seen that $p^{(r)}$ converges to $p$ when $r$ goes to infinity.

2. The Kornya approximation sums to one like a distribution. Hence, (17) and $(19)$ give that

$$
p^{(r)}(0)=\exp \left(-\sum_{n=1}^{r} \frac{\varphi_{p}(n)}{n}\right) .
$$

From $(13)$ and $(21)$, we see that $p^{(r)}(0)$ converges to $p(0)$ when $r$ goes to infinity, if $\sum_{n=1}^{\infty} \varphi_{p}(n) / n$ is convergent. In that case, the proportionality factor between the $r$ th order Kornya approximation and the $r$ th order De Pril approximation converges to one when $r$ goes to infinity. This implies that the $r$ th Kornya approximation converges to infinity when $r$ goes to infinity, as that is the case with the $r$ th order De Pril approximation.

We easily see that for all positive integers $r$ the $r$ th order De Pril approximation of the convolution of distributions in $\mathcal{P}_{0}$ is the convolution of the $r$ th order De Pril approximations of these distributions. Analogous for the Kornya approximation.

Application of (19) in (18) gives the recursion

$$
\begin{aligned}
& P^{(r)}(n)=P^{(r)}(n-1)+ \\
& \frac{1}{n}\left(\sum_{i=1}^{r} \Delta \varphi_{p}(i) P^{(r)}(n-i)-\varphi_{p}(r) P^{(r)}(n-r-1)\right) \cdot(n=1,2, \ldots)
\end{aligned}
$$

From 20, , 19, and 16 , we obtain that when $p^{(r)}$ is the De Pril approximation, then

$$
P^{(r)}(\infty)=p(0) \exp \left(\sum_{n=1}^{r} \frac{\varphi_{p}(n)}{n}\right) .
$$


Hence, $P^{(r)}(\infty)$ can be evaluated recursively by

$$
P^{(r)}(\infty)=P^{(r-1)}(\infty) \exp \left(\frac{\varphi_{p}(r)}{r}\right) \quad(r=1,2, \ldots)
$$

with $P^{(0)}(\infty)=p(0)$.

Now, let us consider an insurance portfolio of $m$ independent policies. For $j=1,2, \ldots, m$, the $j$ th policy has aggregate claims distribution $f_{j} \in \mathcal{P}_{0}$. We want to evaluate the aggregate claims distribution $f=*_{j=1}^{m} f_{j}$ of the portfolio. We express each $f_{j}$ as $f_{j}=p_{j} \vee h_{j}$ where $p_{j}$ is the Bernoulli distribution $\operatorname{Bern}\left(\pi_{j}\right)$ with $\pi_{j}=1-f_{j}(0)$ and $h_{j} \in \mathcal{P}_{+}$given by $h_{j}(y)=f_{j}(y) / \pi_{j}$ for $y=1,2, \ldots$ Then we approximate $p_{j}$ with a function $p_{j}^{(r)} \in \mathcal{F}_{0}^{(r)}$, but keep the severity distribution $h_{j}$ unchanged. Hence, we approximate $f$ with $f^{(r)}=*_{j=1}^{m} f_{j}^{(r)}$ with $f_{j}^{(r)}=p_{j}^{(r)} \vee h_{j}$ for each $j$. We evaluate $f^{(r)}$ by De Pril's second method.

The distribution $p=*_{j=1}^{m} p_{j}$ is the distribution of the number of policies with claims, and we approximate that distribution with $p^{(r)}=*_{j=1}^{m} p_{j}^{(r)}$. The special case when the $p_{j}$ s are approximated with the De Pril approximation, will be studied in Section 6 .

In the present case, insertion of $(8)$ in $(6)$ gives

$$
\varphi_{p}(n)=\sum_{j=1}^{m} \varphi_{p_{j}}(n)=-\sum_{j=1}^{m}\left(\frac{\pi_{j}}{\pi_{j}-1}\right)^{n} .
$$

Thus, for $n=2,3, \ldots$, we obtain

$$
\Delta \varphi_{p}(n)=-\sum_{j=1}^{m} \frac{\pi_{j}^{n-1}}{\left(\pi_{j}-1\right)^{n}} . \quad(n=2,3, \ldots)
$$

When the $p_{j}^{(r)}$ s satisfy $(19)$, then that is also the case with $p^{(r)}$ and insertion of $(24)$ in $(22)$ gives

$$
\begin{gathered}
P^{(r)}(n)=\left(1+\frac{1}{n} \sum_{j=1}^{m} \frac{\pi_{j}}{1-\pi_{j}}\right) P^{(r)}(n-1)+ \\
\frac{1}{n}\left(P^{(r)}(n-r-1) \sum_{j=1}^{m}\left(\frac{\pi_{j}}{\pi_{j}-1}\right)^{r}-\sum_{i=2}^{r} P^{(r)}(n-i) \sum_{j=1}^{m} \frac{\pi_{j}^{i-1}}{\left(\pi_{j}-1\right)^{i}}\right) . \\
(n=1,2, \ldots)
\end{gathered}
$$




\section{Inequalities for the De Pril approximation to the distribution of the number of policies with claims}

We shall now study the distribution $p$ of the number of policies with claims in the insurance portfolio model described in Section 5 .

Let $\alpha_{j}=\pi_{j} /\left(1-\pi_{j}\right)$ for $j=1,2, \ldots, m$. From (8), we then obtain

$$
\varphi_{p_{j}}(n)=(-1)^{n+1} \alpha_{j}^{n} . \quad(n=1,2, \ldots)
$$

For all positive integers $r$, let $p_{j}^{(r)}$ denote the $r$ th order De Pril approximation of $p_{j}$. Then (20) and (19) give

$$
\begin{gathered}
p_{j}^{(r)}(0)=p_{j}(0)=1-\pi_{j} \\
\varphi_{p_{j}^{(r)}}(n)=I(n \leq r) \varphi_{p_{j}}(n)=I(n \leq r)(-1)^{n+1} \alpha_{j}^{n} . \quad(n=1,2, \ldots)
\end{gathered}
$$

We want to approximate the distribution $p=*_{j=1}^{m} p_{j}$ of the number of policies with claims by $p^{(r)}=*_{j=1}^{m} p_{j}^{(r)}$. We have

$$
\begin{gathered}
\varphi_{p}(n)=(-1)^{n+1} \sum_{j=1}^{m} \alpha_{j}^{n} \quad(n=1,2, \ldots) \\
\varphi_{p^{(r)}}(n)=I(n \leq r) \varphi_{p}(n)=I(n \leq r)(-1)^{n+1} \sum_{j=1}^{m} \alpha_{j}^{n}(n=1,2, \ldots) \\
p^{(r)}(0)=p(0)=\prod_{j=1}^{m}\left(1-\pi_{j}\right),
\end{gathered}
$$

and application of $(10)$ gives

$$
\varphi_{P^{(r)}}(n)=1+I(n \leq r) \varphi_{p}(n)=1+I(n \leq r)(-1)^{n+1} \sum_{j=1}^{m} \alpha_{j}^{n}
$$

for $n=1,2, \ldots$ By insertion in (5), we obtain

$$
\begin{aligned}
& P^{(r)}(n)=\frac{1}{n} \sum_{y=1}^{n}\left(1+I(y \leq r) \varphi_{p}(y)\right) P^{(r)}(n-y)= \\
& \frac{1}{n} \sum_{y=1}^{n}\left(1+I(y \leq r)(-1)^{y+1} \sum_{j=1}^{m} \alpha_{j}^{y}\right) P^{(r)}(n-y)(n=1,2, \ldots)
\end{aligned}
$$


with initial value $P^{(r)}(0)=p(0)$.

This immediately gives that

$$
P^{(r)}>0 \quad(r=1,2, \ldots)
$$

when

$$
\varphi_{p} \geq-1
$$

From (25), we see that $\varphi_{p}(2)=-\sum_{j=1}^{m} \alpha_{j}^{2}$. Thus, a necessary condition for (29) is that

$$
\sum_{j=1}^{m} \alpha_{j}^{2} \leq 1
$$

In that case, we must have $\alpha_{j} \leq 1$ for each $j$, and, if this condition is fulfilled, then $\varphi_{p} \geq \varphi_{p}(2)$. Hence, 29$)$ holds if and only if $(30)$ holds.

The following theorem shows that the inequalities

$$
\begin{aligned}
& 0 \leq P^{(2)}(n) \leq P^{(4)}(n) \leq P^{(6)}(n) \leq \cdots \leq P(n) \leq \cdots \leq \\
& P^{(5)}(n) \leq P^{(3)}(n) \leq P^{(1)}(n) .
\end{aligned}
$$

hold for sufficiently large $n$ when $\alpha_{j}<1$ (that is, $\pi_{j}<1 / 2$ ) for all $j$.

Theorem 2. If $\alpha_{j}<1$ for $j=1,2, \ldots, m$, then

$$
\begin{aligned}
& 0<P^{(2)}(\infty)<P^{(4)}(\infty)<P^{(6)}(\infty)<\cdots<P(\infty)<\cdots< \\
& P^{(5)}(\infty)<P^{(3)}(\infty)<P^{(1)}(\infty) .
\end{aligned}
$$

Proof. Insertion of 25) in 23) gives

$$
P^{(r)}(\infty)=P^{(r-1)}(\infty) \exp \left(\frac{(-1)^{r+1}}{r} \sum_{j=1}^{m} \alpha_{j}^{r}\right) . \quad(r=1,2, \ldots)
$$

Hence, for all positive integers $r$,

$$
\begin{aligned}
& P^{(r+2)}(\infty)=P^{(r)}(\infty) \exp \left(\frac{(-1)^{r+2}}{r+1} \sum_{j=1}^{m} \alpha_{j}^{r+1}+\frac{(-1)^{r+3}}{r+2} \sum_{j=1}^{m} \alpha_{j}^{r+2}\right)= \\
& P^{(r)}(\infty) \exp \left(\frac{(-1)^{r}}{(r+1)(r+2)} \sum_{j=1}^{m}\left(1+(r+1)\left(1-\alpha_{j}\right)\right) \alpha_{j}^{r+1}\right) .
\end{aligned}
$$

As $0<\alpha_{j}<1$ for each $j$, we have

$$
\exp \left(\frac{(-1)^{r}}{(r+1)(r+2)} \sum_{j=1}^{m}\left(1+(r+1)\left(1-\alpha_{j}\right)\right) \alpha_{j}^{r+1}\right)\left\{\begin{array}{c}
< \\
>
\end{array}\right\} 1 .\left(r\left\{\begin{array}{c}
\text { odd } \\
\text { even }
\end{array}\right\}\right)
$$


Furthermore, from 12 and 26 follows that $P^{(r)}(\infty)>0$. Hence,

$$
P^{(r+2)}(\infty)\left\{\begin{array}{l}
< \\
>
\end{array}\right\} P^{(r)}(\infty) . \quad\left(r\left\{\begin{array}{c}
\text { odd } \\
\text { even }
\end{array}\right\}\right)
$$

As $P^{(r)}$ converges to $P$, the inequalities 32 hold.

Q.E.D.

In the following, we shall give necessary conditions for (31) to hold for finite $n$.

Theorem 3. If (30) and

$$
\begin{array}{r}
\sum_{j=1}^{m} \alpha_{j}^{r+1}\left(P^{(r+2)}(n-r-1)-\alpha_{j} P^{(r+2)}(n-r-2)\right) \geq 0 \\
(n=1,2, \ldots ; r=2,3, \ldots)
\end{array}
$$

hold, then

$$
0<P^{(2)} \leq P^{(4)} \leq P^{(6)} \leq \cdots \leq P \leq \cdots \leq P^{(5)} \leq P^{(3)} \leq P^{(1)} .
$$

Proof. For any positive integer $r$, we obviously have

$$
I(i \leq r)=I(i \leq r+2) . \quad(i=1,2, \ldots, r-1, r, r+3, r+4, \ldots)
$$

We shall prove by induction on $n$ that

$$
P^{(r+2)}(n) \leq P^{(r)}(n) . \quad(n=0,1,2, \ldots ; r=1,3,5, \ldots)
$$

We have $P^{(r)}(0)=p(0)=P^{(r+2)}(0)$ so the induction hypothesis 36 . holds for $n=0$.

Let us now assume that it holds for $n=1,2, \ldots, k-1$ for some positive integer $k$.

We first assume that $k \leq r$. By application of (27), (29), (35), and (36), we obtain

$$
\begin{aligned}
& P^{(r+2)}(k)=\frac{1}{k} \sum_{i=1}^{k}\left(1+I(i \leq r+2) \varphi_{p}(i)\right) P^{(r+2)}(k-i) \leq \\
& \frac{1}{k} \sum_{i=1}^{k}\left(1+I(i \leq r) \varphi_{p}(i)\right) P^{(r)}(k-i)=P^{(r)}(k),
\end{aligned}
$$

that is, the induction hypothesis (36) holds also for $n=k$.

From (25), we obtain that $\varphi_{p}(r+1)<0$, so that (37) holds also for $k=r+1$, that is, the induction hypothesis (36) holds also for $n=r+1$. 
Now let $k>r+1$. By (25), (33), and (36), we obtain

$$
\begin{aligned}
& \sum_{i=r+1}^{r+2}\left(1+I(i \leq r+2) \varphi_{p}(i)\right) P^{(r+2)}(k-i)= \\
& \sum_{i=r+1}^{r+2}\left(1+(-1)^{i+1} \sum_{j=1}^{m} \alpha_{j}^{i}\right) P^{(r+2)}(k-i)=\sum_{i=r+1}^{r+2} P^{(r+2)}(k-i)- \\
& \sum_{j=1}^{m} \alpha_{j}^{r+1}\left(P^{(r+2)}(k-r-1)-\alpha_{j} P^{(r+2)}(k-r-2)\right) \leq \\
& \sum_{i=r+1}^{r+2} P^{(r+2)}(k-i) \leq \sum_{i=r+1}^{r+2} P^{(r)}(k-i)= \\
& \sum_{i=r+1}^{r+2}\left(1+I(i \leq r) \varphi_{p}(i)\right) P^{(r)}(k-i) .
\end{aligned}
$$

This implies that (37) holds also for $k>r+1$, that is, the induction hypothesis (36) holds also for $n>r+1$.

We have now shown that the induction hypothesis (36) holds for all nonnegative integers $n$. As $P^{(r)}$ converges to $P$, 36 implies that

$$
P \leq \cdots \leq P^{(5)} \leq P^{(3)} \leq P^{(1)} .
$$

The inequalities

$$
P^{(2)} \leq P^{(4)} \leq P^{(6)} \leq \cdots \leq P
$$

are proved analogously.

This completes the proof of Theorem 3 .

The condition (33) is obviously satisfied when

$$
P^{(r)}(n) \geq \alpha_{l} P^{(r)}(n-1) .(n=1,2, \ldots ; r=2,3, \ldots ; l=1,2, \ldots, m)
$$

The following two theorems give sufficient conditions for 30 and 38 to hold.

\section{Theorem 4. If}

$$
\alpha_{l} \leq 1-\sum_{j=1}^{m} \alpha_{j}^{2}, \quad(l=1,2, \ldots, m)
$$

then (30) and (38) hold. 
Proof. From (39), we immediately see that (30) holds and $\alpha_{1}, \alpha_{2}, \ldots, \alpha_{m} \in$ $(0,1)$.

We shall prove by induction on $n$ that (38) holds.

Application of (27) gives that

$$
P^{(r)}(1)-\alpha_{l} P^{(r)}(0)=\left(1+\sum_{j=1}^{m} \alpha_{j}-\alpha_{l}\right) P^{(r)}(0) \geq 0,
$$

so that $(38)$ is satisfied for $n=1$.

Let us now assume that (38) is satisfied for $n=1,2, \ldots, k-1$ for some positive integer $k$. We shall show that it then holds also for $n=k$. Application of (27) gives

$$
\begin{aligned}
& k\left(P^{(r)}(k)-\alpha_{l} P^{(r)}(k-1)\right)=\sum_{i=1}^{k}\left(1+I(i \leq r) \varphi_{p}(i)\right) P^{(r)}(k-i)- \\
& \alpha_{l}\left(P^{(r)}(k-1)+\sum_{i=1}^{k-1}\left(1+I(i \leq r) \varphi_{p}(i)\right) P^{(r)}(k-1-i)\right)= \\
& \left(1-\alpha_{l}\right) \sum_{i=1}^{k} P^{(r)}(k-i)+\sum_{i=1}^{r} \varphi_{p}(i)\left(P^{(r)}(k-i)-\alpha_{l} P^{(r)}(k-1-i)\right) \geq \\
& \left(1-\alpha_{l}\right) P^{(r)}(k-2)+\sum_{i=1}^{r} \varphi_{p}(i)\left(P^{(r)}(k-i)-\alpha_{l} P^{(r)}(k-1-i)\right)= \\
& \left(1-\alpha_{l}\right) P^{(r)}(k-2)+\varphi_{p}(1) P^{(r)}(k-1)+ \\
& \sum_{i=2}^{r}\left(\varphi_{p}(i)-\alpha_{l} \varphi_{p}(i-1)\right) P^{(r)}(k-i)-\alpha_{l} \varphi_{p}(r) P^{(r)}(k-1-r)= \\
& \left(1-\alpha_{l}\right) P^{(r)}(k-2)+\varphi_{p}(1) P^{(r)}(k-1)+\left(\varphi_{p}(2)-\alpha_{l} \varphi_{p}(1)\right) P^{(r)}(k-2)+ \\
& \sum_{i=3}^{r}\left(\varphi_{p}(i)-\alpha_{l} \varphi_{p}(i-1)\right) P^{(r)}(k-i)-\alpha_{l} \varphi_{p}(r) P^{(r)}(k-1-r)= \\
& \left(1-\alpha_{l}+\varphi_{p}(2)\right) P^{(r)}(k-2)+\varphi_{p}(1)\left(P^{(r)}(k-1)-\alpha_{l} P^{(r)}(k-2)\right)+ \\
& \sum_{i=3}^{r}\left(\varphi_{p}(i)-\alpha_{l} \varphi_{p}(i-1)\right) P^{(r)}(k-i)-\alpha_{l} \varphi_{p}(r) P^{(r)}(k-1-r) .
\end{aligned}
$$


Insertion of (25) gives

$$
\begin{aligned}
& k\left(P^{(r)}(k)-\alpha_{l} P^{(r)}(k-1)\right) \geq\left(1-\alpha_{l}-\sum_{j=1}^{m} \alpha_{j}^{2}\right) P^{(r)}(k-2)+ \\
& \left(P^{(r)}(k-1)-\alpha_{l} P^{(r)}(k-2)\right) \sum_{j=1}^{m} \alpha_{j}+\sum_{j=1}^{m} S_{k j l}^{(r)}
\end{aligned}
$$

with

$$
S_{k j l}^{(r)}=\left(\alpha_{l}+\alpha_{j}\right) \sum_{i=3}^{k_{r}}\left(-\alpha_{j}\right)^{i-1} P^{(r)}(k-i)+\alpha_{l}\left(-\alpha_{j}\right)^{r} P^{(r)}(k-1-r)
$$

with $k_{r}=\min (r, k)$.

By (39), 28), and the induction hypothesis (38), the first two terms in (40) are non-negative so that

$$
P^{(r)}(k)-\alpha_{l} P^{(r)}(k-1) \geq \frac{1}{k} \sum_{j=1}^{m} S_{k j l}^{(r)}
$$

We immediately see that $S_{k j l}^{(2)} \geq 0$.

For $r>2$, we obtain

$$
\begin{aligned}
& S_{k j l}^{(r)}=\left(\alpha_{l}+\alpha_{j}\right) \times \\
& \left(\sum_{i=2}^{\left[k_{r} / 2\right]} \alpha_{j}^{2 i-2}\left(P^{(r)}(k-2 i+1)-\alpha_{j} P^{(r)}(k-2 i)\right)+I\left(k_{r} \text { odd }\right)\left(-\alpha_{j}\right)^{k_{r}-1} P^{(r)}\left(k-k_{r}\right)\right)+ \\
& \alpha_{l}\left(-\alpha_{j}\right)^{r} P^{(r)}(k-1-r) .
\end{aligned}
$$

By the induction hypothesis (38), this gives

$S_{k j l}^{(r)} \geq$

$I\left(k_{r}\right.$ odd $)\left(\alpha_{l}+\alpha_{j}\right)\left(-\alpha_{j}\right)^{k_{r}-1} P^{(r)}\left(k-k_{r}\right)+\alpha_{l}\left(-\alpha_{j}\right)^{r} P^{(r)}(k-1-r)$.

When $k \leq r$, the last term vanishes and $k_{r}=k$, so that

$$
S_{k j l}^{(r)} \geq I(k \text { odd })\left(\alpha_{l}+\alpha_{j}\right)\left(-\alpha_{j}\right)^{k-1} P^{(r)}(0) \geq 0 .
$$

Let us now turn to the case when $k>r$. Then $k_{r}=r$, and insertion in (42) gives

$$
S_{k j l}^{(r)} \geq I(r \text { odd })\left(\alpha_{l}+\alpha_{j}\right)\left(-\alpha_{j}\right)^{r-1} P^{(r)}(k-r)+\alpha_{l}\left(-\alpha_{j}\right)^{r} P^{(r)}(k-1-r) .
$$


When $r$ is even, the first term vanishes, and the last term is non-negative so that $S_{k j l}^{(r)} \geq 0$. When $r$ is odd, we obtain

$$
\begin{aligned}
& S_{k j l}^{(r)} \geq\left(\alpha_{l}+\alpha_{j}\right)\left(-\alpha_{j}\right)^{r-1} P^{(r)}(k-r)+\alpha_{l}\left(-\alpha_{j}\right)^{r} P^{(r)}(k-1-r)= \\
& \left(-\alpha_{j}\right)^{r-1}\left(\alpha_{l} P^{(r)}(k-r)+\alpha_{j}\left(P^{(r)}(k-r)-\alpha_{l} P^{(r)}(k-1-r)\right)\right) \geq 0
\end{aligned}
$$

by the induction hypothesis (38).

We have now shown that we always have $S_{k j l}^{(r)} \geq 0$. Insertion in 41 gives that the induction hypothesis (38) is satisfied when $n=k$, and by induction follows that it is satisfied for all positive integers $n$.

This completes the proof of Theorem 4 .

Theorem 5. If (30) and

$$
\alpha_{l} \leq\left(\sum_{j=1}^{m} \alpha_{j}+1\right)^{-1} \quad(l=1,2, \ldots, m)
$$

hold, then (38) holds.

Proof. From (43), we see that $\alpha_{1}, \alpha_{2}, \ldots, \alpha_{m} \in(0,1)$, and (30) gives that (28) holds. We shall prove by induction on $n$ that (38) holds.

Analogous to the proof of Theorem 4, we obtain that (38) holds for $n=1$.

Let us now assume that (38) holds for $n=1,2, \ldots, k-1$ for some integer $k>1$. We shall show that it also holds for $n=k$. The deduction of (40) is also valid under the present assumptions. We obtain

$$
\begin{aligned}
& \left(1-\alpha_{l}-\sum_{j=1}^{m} \alpha_{j}^{2}\right) P^{(r)}(k-2)+\left(P^{(r)}(k-1)-\alpha_{l} P^{(r)}(k-2)\right) \sum_{j=1}^{m} \alpha_{j}= \\
& \left(1-\alpha_{l}\left(1+\sum_{j=1}^{m} \alpha_{j}\right)\right) P^{(r)}(k-2)+ \\
& \sum_{j=1}^{m} \alpha_{j}\left(P^{(r)}(k-1)-\alpha_{j} P^{(r)}(k-2)\right) \geq 0
\end{aligned}
$$

by (43) and the induction hypothesis (38). Hence, (41) still holds, and, analogous to the proof of Theorem 4, we obtain that the induction hypothesis (38) holds also for $n=k$. By induction follows that it holds for all positive integers $n$.

This completes the proof of Theorem 5 Q.E.D. 


\section{$7 \quad$ Possible further results}

In Section 6, we gave sufficient conditions for the ordering (34) to hold for the distribution of the number of policies with claims in an insurance portfolio of independent policies when we use the De Pril approximation. As pointed out in Section 1, we hope these results can be step-stones for deducing more general results.

A natural question is now whether it would be possible to extend our results to give sufficient conditions for the ordering

$$
0 \leq F^{(2)} \leq F^{(4)} \leq F^{(6)} \leq \cdots \leq F \leq \cdots \leq F^{(5)} \leq F^{(3)} \leq F^{(1)}
$$

in the insurance portfolio model of Section 5. For such extension, perhaps one should start with the individual life model, that is, each policy can have at most one claim and the size of that claim is fixed.

Many of the error bounds that have been introduced for such approximations, depend on only the Bernoulli parameters, not the severity distributions. Could this be the case also for sufficient conditions for the ordering (44)? At least, Theorem 1 immediately gives that $F^{(r)}(\infty)=P^{(r)}(\infty)$ for all $r$, so that

$$
\begin{aligned}
& 0<F^{(2)}(\infty)<F^{(4)}(\infty)<F^{(6)}(\infty)<\cdots<F(\infty)<\cdots< \\
& F^{(5)}(\infty)<F^{(3)}(\infty)<F^{(1)}(\infty)
\end{aligned}
$$

if and only if (32) holds.

As pointed out in Section 1, when using the Kornya approximation instead of the De Pril approximation, numerical examples often indicate the ordering

$$
0 \leq \tilde{F}^{(1)} \leq \tilde{F}^{(3)} \leq \tilde{F}^{(5)} \leq \cdots \leq F \leq \cdots \leq \tilde{F}^{(6)} \leq \tilde{F}^{(4)} \leq \tilde{F}^{(2)},
$$

where we have added a tilde to distinguish from the De Pril approximation.

As the $r$ th order Kornya approximation is proportional with the $r$ th order De Pril approximation, we have

$$
F^{(r)}(\infty)=\frac{F^{(r)}(\infty)}{\tilde{F}^{(r)}(\infty)}=\frac{F^{(r)}(0)}{\tilde{F}^{(r)}(0)}=\frac{F(0)}{\tilde{F}^{(r)}(0)}
$$

Thus,

$$
\begin{aligned}
& \tilde{F}^{(1)}(0)<\tilde{F}^{(3)}(0)<\tilde{F}^{(5)}(0)<\cdots<F(0)<\cdots< \\
& \tilde{F}^{(6)}(0)<\tilde{F}^{(4)}(0)<\tilde{F}^{(2)}(0)
\end{aligned}
$$


if and only if

$$
\begin{aligned}
& F^{(2)}(\infty)<F^{(4)}(\infty)<F^{(6)}(\infty)<\cdots<F(\infty)<\cdots< \\
& F^{(5)}(\infty)<F^{(3)}(\infty)<F^{(1)}(\infty) .
\end{aligned}
$$

It would have been very nice if the ordering (45) could hold under the same conditions as the corresponding ordering for the De Pril approximation. Unfortunately, in the numerical example of Section 8, Theorem 3 gives that the ordering for the De Pril approximation holds, but numerical calculations show that the ordering for the Kornya approximation does not hold. The question is then whether it would be possible to find conditions under which both (44) and (45) hold. That would be very convenient as the approximations of De Pril and Kornya are proportional, so that we could obtain upper and lower bounds for $P$ by using the same approximation and a scaling factor.

If both (44) and (45) hold, then we have

$$
(-1)^{r} F^{(r)} \leq(-1)^{r} F \leq(-1)^{r} \tilde{F}^{(r)} . \quad(r=1,2, \ldots)
$$

By using the proportionality between the $r$ th order Kornya approximation and the $r$ th order De Pril approximation, we obtain

$$
(-1)^{r} \leq(-1)^{r} \frac{F}{F^{(r)}} \leq(-1)^{r} \kappa^{(r)} \quad(r=1,2, \ldots)
$$

with

$$
\kappa^{(r)}=\frac{\tilde{F}^{(r)}(0)}{F^{(r)}(0)}=\frac{\tilde{f}^{(r)}(0)}{f^{(r)}(0)}=\prod_{j=1}^{m} \frac{\tilde{f}_{j}^{(r)}(0)}{f_{j}^{(r)}(0)}=\prod_{j=1}^{m} \frac{\tilde{p}_{j}^{(r)}(0)}{p_{j}^{(r)}(0)} .
$$

Application of (21), (20), and (8) gives

$$
\begin{gathered}
\kappa^{(r)}=\prod_{j=1}^{m} \frac{\exp \left(\sum_{y=1}^{r} \frac{1}{y}\left(\frac{\pi_{j}}{\pi_{j}-1}\right)^{y}\right)}{1-\pi_{j}}= \\
\prod_{j=1}^{m} \frac{\exp \left(\sum_{y=1}^{r}\left(1-f_{j}(0)^{-1}\right)^{y} / y\right)}{f_{j}(0)} .
\end{gathered}
$$

\section{Numerical example}

As a numerical example, we study a life assurance portfolio introduced by Gerber (1979). The portfolio consists of 31 independent policies. Each policy can have at most one claim. Thus, the number of policies with claims is the 


\begin{tabular}{|r|c|c|ccccc|r|}
\hline & & & \multicolumn{7}{|c|}{ Amount } & \\
\cline { 4 - 7 }$j$ & $\pi_{j}$ & $\alpha_{j}$ & 1 & 2 & 3 & 4 & 5 & $k_{j}$ \\
\hline 1 & 0.03 & 0.030928 & 2 & 3 & 1 & 2 & 0 & 8 \\
2 & 0.04 & 0.041667 & 0 & 1 & 2 & 2 & 1 & 6 \\
3 & 0.05 & 0.052632 & 0 & 2 & 4 & 2 & 2 & 10 \\
4 & 0.06 & 0.063830 & 0 & 2 & 2 & 2 & 1 & 7 \\
\hline
\end{tabular}

Table 1: Gerber's portfolio.

number of claims. Furthermore, the claims have fixed amounts. There are $m=4$ different claim probabilities and five different claim amounts. As we are going to concentrate on the distribution $p$ of the number of claims, the amounts are of less interest to us. For $j=1,2, \ldots, m$, we let $\pi_{j}$ denote the $j$ th claim probability and $k_{j}$ the number of policies with this claim probability. We also introduce $\alpha_{j}=\pi_{j} /\left(1-\pi_{j}\right)$ for $j=1,2, \ldots, m$.

In Table 1, we display the number of policies for each combination of amount and claim probability, as well as the $\alpha_{j}$ s and the $k_{j}$ s.

We now have

$$
1-\sum_{j=1}^{m} k_{j} \alpha_{j}^{2}=0.925711,
$$

which is much greater than the $\alpha_{j} \mathrm{~s}$, so that (39) is fulfilled. Hence, the ordering (34) holds.

In Table 2, we display the exact tail $\bar{P}$ of the claim number distribution, as well as the De Pril approximation of order 1, 2, 3, and 4. As expected, these figures satisfy the ordering (44).

The corresponding calculations with the De Pril approximation replaced with the Kornya approximation are shown in Table 3. These figures do not satisfy the ordering (45); in particular, $P^{(4)}(n)>P^{(2)}(n)$ for $n=$ $15,16,17,18$.

As we wanted to study the ordering properties far out in the tail, we have not used the recursive methods with their risk of error accumulation, but rather power series expansion of (15) with the MuPad engine of Scientific Workplace, version 5.5. As a control, we did the same calculations with the Maple engine of Scientific Workplace, version 3.0. The calculations were reasonably consistent at least up to $n=20$.

In Table 4, we display the proportionality factor

$$
\kappa^{(r)}=\prod_{j=1}^{m}\left(\frac{\exp \left(\sum_{y=1}^{r} \frac{1}{y}\left(\frac{\pi_{j}}{\pi_{j}-1}\right)^{y}\right)}{1-\pi_{j}}\right)^{k_{j}} .
$$




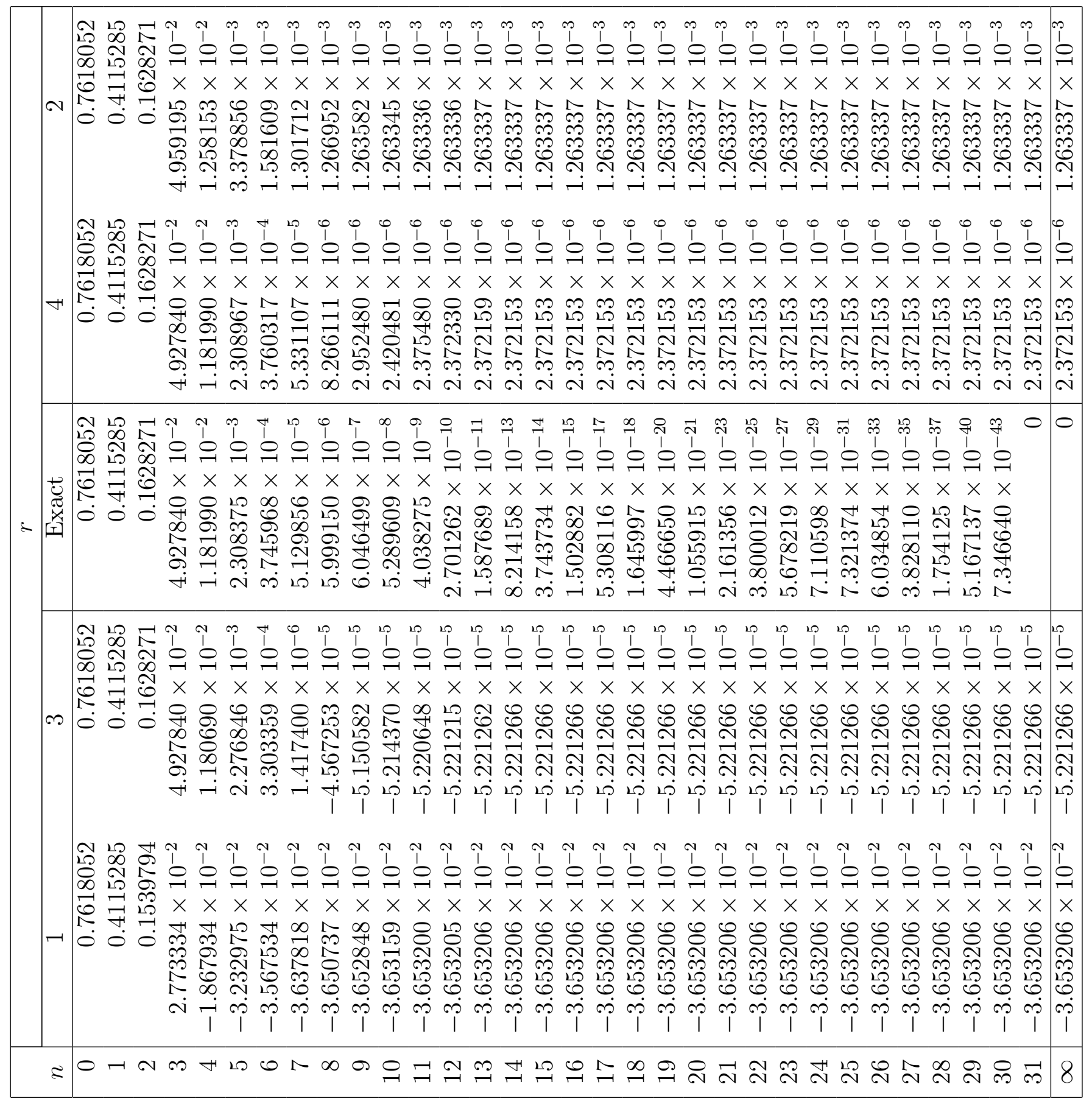




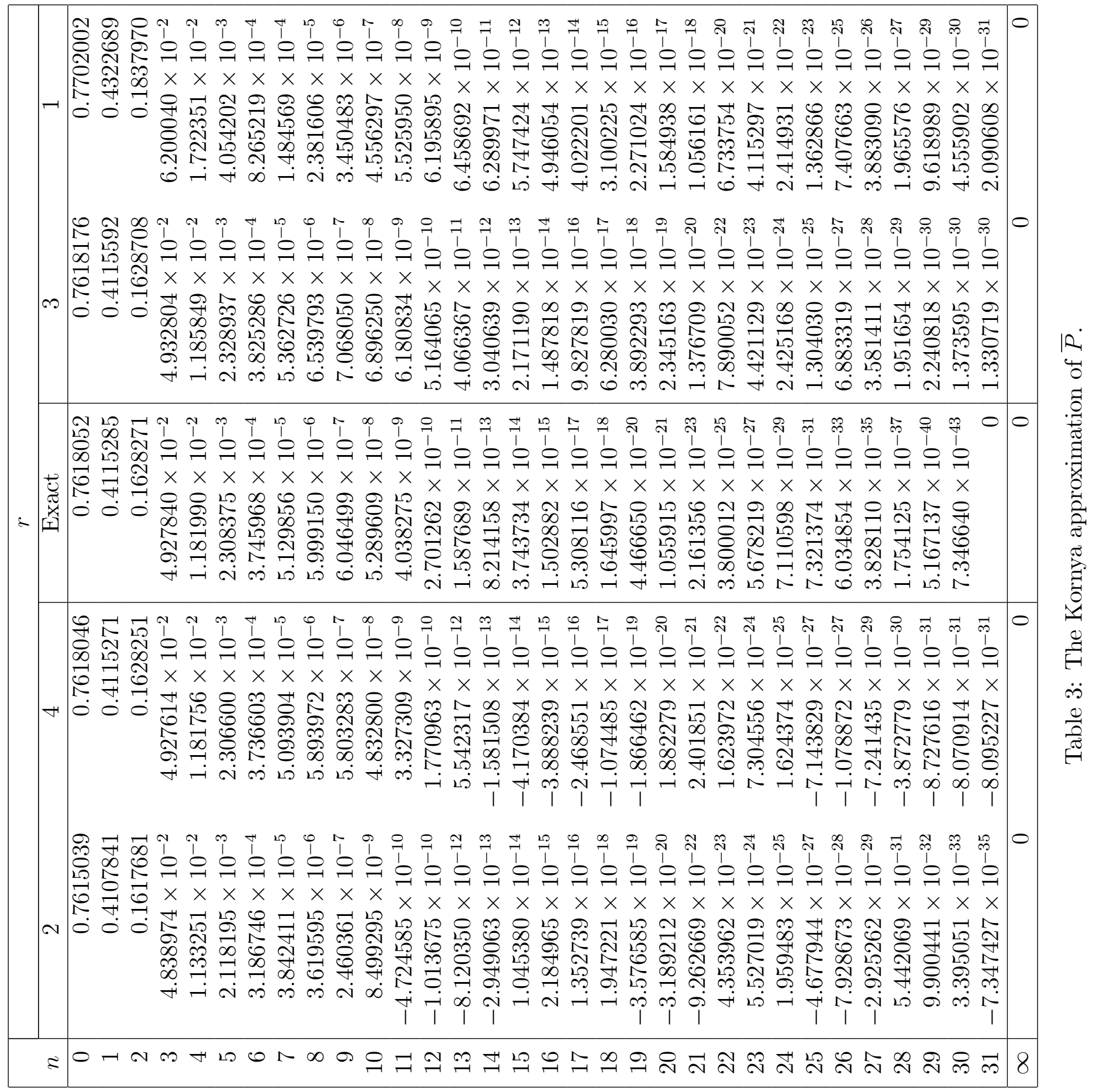




\begin{tabular}{|c|cccc|}
\hline$r$ & 1 & 2 & 3 & 4 \\
\hline$\kappa^{(r)}$ & 0.9647555 & 1.0012649 & 0.9999478 & 1.0000024 \\
\hline
\end{tabular}

Table 4: The proportionality factor.

Calculations for the aggregate claims distribution presented by Dhaene \& Vandebroek (1988) and Sundt \& Vernic (2009) satisfy the orderings (44) and (45).

\section{References}

Chan, F.Y. (1984). On a family of aggregate claims distributions. Insurance: Mathematics and Economics 3, 151-155.

De Pril, N. (1986). On the exact computation of the aggregate claims distribution in the individual life model. ASTIN Bulletin 16, 109-112.

De Pril, N. (1988). Improved approximations for the aggregate claims distribution of a life insurance portfolio. Scandinavian Actuarial Journal, 61-68.

De Pril, N. (1989). The aggregate claims distribution in the individual model with arbitrary positive claims. ASTIN Bulletin 19, 9-24.

Dhaene, J. \& De Pril, N. (1994). On a class of approximative computation methods in the individual model. Insurance: Mathematics and Economics 14, 181-196.

Dhaene, J. \& Goovaerts, M.J. (1997). On the dependency of risks in the individual life model. Insurance: Mathematics and Economics 19, 243-253.

Dhaene, J., Ribas, C., \& Vernic, R. (2006). Recursions for the individual risk model. Acta Mathematicae Applicatae Sinicae, English Series, 14, 543-564.

Dhaene, J. \& Sundt, B. (1998). On approximating distributions by approximating their De Pril transforms. Scandinavian Actuarial Journal, 1-23.

Dhaene, J., Willmot, G.E., \& Sundt, B. (1999). Recursions for distribution functions and their stop-loss transforms. Scandinavian Actuarial Journal, 52-65. Corrigendum. Scandinavian Actuarial Journal (2007), 226.

Gerber, H.U. (1979). An introduction to mathematical risk theory. Huebner Foundation Monographs 8, Richard D. Irwin, Inc., Homewood, Illinois.

Hipp, C. (1986). Improved approximations for the aggregate claims distribution in the individual model. ASTIN Bulletin 16, 89-100. 
Jewell, W.S. \& Sundt, B. (1981). Improved approximations for the distribution of a heterogeneous risk portfolio. Bulletin of the Association of Swiss Actuaries, 221-240.

Kornya, P.S. (1983). Distribution of aggregate claims in the individual risk theory model. TSA 35, 823-836. Discussion. Transactions of the Society of Actuaries 35, 837-858.

Kornya, P.S. (2007). On approximating the individual risk model. North American Aactuarial Journal 11, 77-98.

Kuon, S., Radtke, M., \& Reich, A. (1993). An appropriate way to switch from the individual risk model to the collective one. ASTIN Bulletin 23, $23-54$.

Sundt, B. (1985). On approximations for the distribution of a heterogeneous risk portfolio. Bulletin of the Association of Swiss Actuaries, 189-203.

Sundt, B. (1995). On some properties of De Pril transforms of counting distributions. ASTIN Bulletin 25, 19-31.

Sundt, B. (2002). Recursive evaluation of aggregate claims distributions. Insurance: Mathematics and Economics 30, 297-323.

Sundt, B. \& Vernic, R. (2006). A binomial method for evaluating the aggregate claims distribution in De Pril's individual risk model. Belgian Actuarial Bulletin 6, 5-13.

Sundt, B. \& Vernic, R. (2009). Recursions for convolutions and compound distributions with insurance applications. Springer-Verlag, Heidelberg. To appear.

Vandebroek, M. \& De Pril, N. (1988). Recursions for the distribution of a life portfolio: A numerical comparison. Bulletin of the Royal Association of Belgian Actuaries 82, 21-35.

Raluca Vernic

Faculty of Mathematics and Computer Science

Ovidius University of Constanta

124 Mamaia Blvd

8700 Constanta

ROMANIA 
Jan Dhaene

Centre for Risk and Insurance Studies

Katholieke Universiteit Leuven

Naamsestraat 69 - bus 3507

3000 Leuven

BELGIUM

Bjørn Sundt

Karenslyst allé $1 \mathrm{~A}$

P.O. Box 1380 Vika

0278 Oslo

NORWAY 